\title{
Not so rare: It's time to raise awareness of rare diseases in South Africa
}

There are more than 6000 diseases that individually affect fewer than 1 in 2000 people. The individual prevalence of these 'rare diseases' may be low, but the estimated combined impact on the healthcare system and society is substantial, with an estimated $6-8 \%$ of the population being affected. ${ }^{[1,2]}$ Approximately $80 \%$ of rare diseases are genetic and many are multisystemic. Most are chronic, lifelong, progressive and severely disabling, associated with significant impairment of physical and/or mental ability, reduced quality of life and shortened life expectancy. ${ }^{[3]}$

Globally, and particularly in Africa, rare diseases present a challenge to the healthcare community. Because they are uncommon, expertise is limited and healthcare practitioners lack the awareness and knowledge required for accurate diagnosis, timely referral and appropriate management. Patients remain undiagnosed, are misdiagnosed, or are diagnosed only after many years of illness. ${ }^{[2,3]}$ Socioeconomic status, family life, opportunities for education and employment, and participation in society are all affected, trapping the patient in a vicious cycle of vulnerability and poverty.

Even if their condition is correctly identified, people living with a rare disease face numerous challenges in accessing treatment, healthcare and social care. In South Africa (SA), rare diseases are seldom a consideration when it comes to healthcare planning and policy-making. Furthermore, patients are frequently neglected or dismissed by healthcare funders, delaying treatment or leaving them without access to treatment altogether. Considering that these chronic, life-threatening conditions should be covered in full under Prescribed Minimum Benefits (PMB), regardless of the selected plan option, this is an inexcusable practice. Matters are worse in the public sector, where resources are limited, medicines are unavailable, and rare diseases are often ignored altogether. There is an urgent need in SA for more specialised clinics and practitioners equipped to manage these conditions.

Nevertheless, specialised multidisciplinary academic units with the expertise and experience required to assess and manage these patients properly do exist, and it is important to raise awareness of this. These units are equipped to address the unique needs of patients, provide optimal outpatient services, and provide the infrastructure and patient numbers required for further research into the disease and potential management strategies. Patients with a rare disease should be referred to these units, and not treated by individual inexperienced practitioners who only see one or two such patients in their lifetime. The units are open for training to anyone with an interest who wishes to gain experience, including doctors in private practice.

In addition, a number of other important issues need to be addressed. We need to raise awareness among both medical practitioners and society in general about rare diseases, recognition, available information resources, and where to refer for advanced diagnosis and treatment. Collaboration within SA and with international colleagues will facilitate exchange of best practices for clinical care and opportunities for research, and promote utilisation of existing rare disease resources and dissemination channels from government organisations, private sector resources and patient advocacy groups. ${ }^{[3]}$ Clinicians and policy makers need to engage with the pharmaceutical industry to seek solutions to the exorbitantly high cost of medicines, most of which, without the support of funders, are unaffordable to the majority of patients. But we must also think beyond the healthcare system to ensure non-discrimination of affected individuals and their access to education, employment and healthy participation in society.

In this issue of $S A M J$, we describe pretreatment characteristics of a subset of the SA cohort of patients enrolled into the International Collaborative Gaucher Group (ICGG) Gaucher Registry. This inherited autosomal recessive metabolic disorder has a prevalence in the general population of approximately 1 per $60000 .{ }^{[4]}$ We hope that in doing so, we will raise awareness not only of this condition but of rare diseases in general, and the plight of the unfortunate, vulnerable and often marginalised patients who suffer from them.

\section{B F Jacobson (iD}

Departments of Molecular Medicine and Haematology and Internal Medicine, Faculty of Health Sciences, University of the Witwatersrand, Johannesburg, South Africa

clot@nhls.ac.za

\footnotetext{
1. European Organisation for Rare Diseases (EURORDIS). Rare diseases: Understanding this public health priority. November 2005. https://www.eurordis.org/IMG/pdf/princeps_document-EN.pd (accessed 26 March 2021)

2. NGO Committee for Rare Diseases. Report from Rare Diseases Day policy event at the United Nations May 2019. https://www.ngocommitteerarediseases.org (accessed 26 March 2021).

Baynam GS, Groft S, van der Westhuizen FH, et al. A call for global action for rare diseases in Africa. Nat Genet 2020;52(1):21-26. https://doi.org/10.1038/s41588-019-0552-2

Stirnemann J, Belmatoug N, Camou F, et al. A review of Gaucher disease pathophysiology, clinical presentation and treatments. Int J Mol Sci 2017;18:441. https://doi.org/10.3390/ijms18020441
}

S Afr Med J 2022;112(1):6. https://doi.org/10.7196/SAMJ.2022.v112i1.16010 